\title{
Output Feedback control of time delay systems with adaptation of delay estimate
}

\author{
D. Bresch-Pietri ${ }^{*}$ J. Chauvin ${ }^{* *}$ N. Petit ${ }^{* * *}$ \\ * MINES ParisTech, Centre Automatique et Systèmes, Unité Mathématiques et \\ Systèmes, 60 Bd St-Michel, 75272 Paris, Cedex 06, France \\ (e-mail : delphine.bresch-pietri@mines-paristech.fr) \\ ** IFP Energies Nouvelles, Département Contrôle, Signal et Système, 1 et 4 \\ avenue du Bois-Préau, 92852 Rueil-Malmaison, France \\ *** MINES ParisTech, Centre Automatique et Systèmes, Unité Mathématiques \\ et Systèmes, 60 Bd St-Michel, 75272 Paris, Cedex 06, France
}

\begin{abstract}
This paper extends recent results for the literature which employ a backstepping transformation under the form of an infinite dimensional system to address robust control of time delay systems. The contribution treats the case of output feedback through a state observer, while allowing online adaptation of the delay system. A theoretical result is proven and an example illustrates the approach.
\end{abstract}

\section{INTRODUCTION}

This paper addresses the general problem of the equilibrium regulation, through an output feedback, of a potentially unstable linear systems with an unknown input time-delay. As established in numerous recent surveys and research papers (e.g. Richard [2003], Huang and Lin [1995] or Zhong [2006]), the lack of robustness of Smith predictors [1959] with respect to the uncertainty on the delay is still a major concern, especially in view of implementations, in which it often appears as a performance bottleneck.

Lately (see Krstic [2008],Krstic and Bresch-Pietri [2009],Krstic and Smyshlyaev [2008]), new techniques have been proposed to address this uncertainty for (single) input time-delay systems. We follow this methodology and extend it to the case where the system state is not measured. The incorporation in the strategy of a state observer, and the analysis, through a convergence study, of its compliance with different delay adaptation laws are the main contributions of this paper.

In details, the approach we follow is based on a form of backstepping boundary control for partial differential equations (PDEs), modeling the actuator delay as a transport process. This transformation enables the use of systematic Lyapunov tools for establishing stability properties. While the observer design we use could seem classical, the main result stated here and its proof are, up to our knowledge, new.

The main result (Theorem 1) addresses the case of delay adaptation, which satisfy Assumption 4 presented below, requiring the employed update law to improve the delay estimation. It is consistent with numerous delay identification techniques presented for example in O'Dwyer [2000].

The paper is organized as follows. In Section 2, we describe the general framework of the problem under consideration, before presenting in Section 3 the output-feedback strategy we propose. A proof of the convergence properties of this control is provided in Section 4. Finally, in Section 5, we illustrate the merits of our approach through an example from the literature. For this case-study, we consider a delay update law based on a Least Mean-Square method, compliant with the delay estimation assumption stated previously and with on-line implementation.

\section{PROBLEM STATEMENT AND NOTATIONS}

\subsection{Notations}

In the following, $n$ and $p$ are strictly positive integers, $|$.$| refers$ to the usual Euclidean norm whereas the norm $\|$.$\| is defined$ for a functional $f(., t)$ as

$$
\|f(t)\|=\sqrt{\int_{0}^{1} f(x, t)^{2} d x}, f:(x, t) \in[0 ; 1] \times \mathbb{R}_{+} \rightarrow \mathbb{R}
$$

\subsection{Problem formulation}

Consider the system with unknown input time delay

$$
a(s) Y(s)=b(s) e^{-D s} U(s)
$$

where $D>0$ is an unknown positive constant denoting the delay, $s \in \mathbb{C}$ denotes the Laplace variable and $a(s)$ and $b(s)$ are polynomial functions of $s$. One can transform this system into the following state-space realization (see Kailath [1980] for the achievement and the choice of such a formulation), that we consider from now on to design the control

$$
\left\{\begin{array}{l}
\dot{X}(t)=A X(t)+B U(t-D) \\
Y(t)=C X(t)
\end{array}\right.
$$

where $\mathrm{Y} \in \mathbb{R}^{p}$ is the measured output, $X \in \mathbb{R}^{n}$ is unmeasured and $U$ is the scalar input. The following assumptions further characterize the class of considered systems.

Assumption 1. An upper bound $\bar{D}$ and a lower bound $\underline{D}>0$ of the unknown delay $\underline{D} \leq D \leq \bar{D}$ are known.

Assumption 2. The pair $(A, B)$ is controllable, the pair $(A, C)$ is observable, and $(K, L) \in \mathbb{R}^{1 \times n} \times \mathbb{R}^{n \times p}$ are stabilizing gains.

Assumption 3. For a constant set-point $Y^{r}$, there exists a couple $\left(X^{r}, U^{r}\right)$ satisfying

$$
\left\{\begin{array}{l}
0=A X^{r}+B U^{r} \\
Y^{r}=C X^{r}
\end{array}\right.
$$


The existence of such a non-degenerate equilibrium $Y^{r}$ implies that the transfer function does not introduce pure integrators.

The control objective is to have system (2) to track the given set-point $Y^{r}$ through an observer feedback. Before presenting the proposed control design, one can discuss the assumptions listed above. Assumption 1 is formulated for Lyapunov analysis purposes only, and is easily borne out for practical purposes, while Assumption 3 guarantees the well-posedness of the studied problem. Finally, only Assumption 2 can be considered as restrictive. It requires the equivalent delay-free form of the state-space formulation to be both controllable and observable, which is a reasonable assumption to design an output feedback stabilizing controller.

\section{CONTROL DESIGN}

We now present the control method we propose. Following the approach of Krstic [2008] (see also Bresch-Pietri et al. [2010] and Bresch-Pietri and Krstic [2009]), one can introduce a transport model of the delay by defining the distributed input $u(x, t)=U(t+D(x-1)), x \in[0,1]$. The plant (3) can then be represented under the form

$$
\begin{cases}\dot{X}(t) & =A X(t)+B u(0, t) \\ D u_{t}(x, t) & =u_{x}(x, t) \\ u(1, t) & =U(t) \\ Y(t) & =C X(t)\end{cases}
$$

Unfortunately, because the speed of propagation of the distributed waiting line $1 / D$ is uncertain, one cannot deduce the value of $u(x, t)$ for each $x \in[0,1]$ from the history of the given input $U(t)$. Therefore, to design the control, we consider an estimate

$$
\hat{u}(x, t)=U(t+\hat{D}(t)(x-1))
$$

of the distributed input, where $\hat{D}(t)$ is a (varying) estimate of the delay. With this estimate waiting line, we define an observer of the system state

$$
\begin{cases}\dot{\hat{X}}(t) & =A \hat{X}(t)+B \hat{u}(0, t)-L(Y-C \hat{X}(t)) \\ \hat{D}(t) \hat{u}_{t}(x, t) & =\hat{u}_{x}(x, t)+\hat{\hat{D}}(t)(x-1) \hat{u}_{x}(x, t) \\ \hat{u}(1, t) & =U(t)\end{cases}
$$

When both the system state and the delay value are perfectly known, the following predictive controller (see e.g. Smith [1959], Artstein [1982]) achieves exponential stability of system (3) toward 0

$$
\begin{aligned}
U(t) & =K X_{P}(t+D) \\
& =K\left(e^{A D} X(t)+\int_{t-D}^{t} e^{A(t-s)} B U(s) d s\right)
\end{aligned}
$$

This controller can be understood as a delay-version of the classical proportional controller, where $X_{P}(t+D)$ can be interpreted as a $D$-units of time ahead prediction of the system state, starting from $X(t)$ and driven by the control history over a $D$-units of time window. Applying the certainty equivalence principle, we use here the control law

$$
\begin{aligned}
U(t)= & U^{r}-K X^{r}+K\left[e^{A \hat{D}(t)} \hat{X}(t)\right. \\
& \left.+\hat{D}(t) \int_{0}^{1} e^{A \hat{D}(t)(1-y)} B \hat{u}(y, t) d y\right]
\end{aligned}
$$

where the estimate delay satisfies the following "growth condition".
Assumption 4. There exists a positive constant $M>0$ such that, with $\tilde{D}(t)=D-\hat{D}(t)$ and $\tau_{D} \in \mathscr{C}^{0}([0 ;+\infty[)$,

$$
\begin{gathered}
\dot{\hat{D}}(t)=\gamma_{D} \operatorname{Proj}_{[\underline{D} ; \bar{D}]}\left\{\tau_{D}(t)\right\} \\
\forall t \geq 0, \tau_{D}(t) \tilde{D}(t) \geq 0 \text { and }\left|\tau_{D}(t)\right|<M
\end{gathered}
$$

This assumption allows one to consider sharp delay update laws, provided that the direction $\tau_{D}$ improves the estimate. This can be achieved using various methods (for a survey of these methods see O'Dwyer [2000]), exemplified in this article in Section 5. Finally, for sake of conciseness, we introduce the following error variables used below

$$
\begin{aligned}
\Delta X(t) & =X(t)-X^{r} \\
\Delta \hat{X} & =\hat{X}(t)-X^{r} \\
e(x, t) & =u(x, t)-U^{r} \\
\hat{e}(x, t) & =\hat{u}(x, t)-U^{r} \\
\tilde{X}(t) & =X(t)-\hat{X}(t) \\
\tilde{e}(x, t) & =e(x, t)-\hat{e}(x, t)
\end{aligned}
$$

where (12)-(15) represent the tracking errors and (16)-(17) the estimation errors, resulting, respectively, from the observation of the state and of the estimation of the waiting line.

Theorem 1. Consider the closed-loop system consisting of (5), the control law (9), the estimate plant (7) with a delay update law satisfying Assumption 4. Let us define

$$
\begin{aligned}
\Gamma(t)= & |\Delta X(t)|^{2}+|\Delta \hat{X}(t)|^{2}+\|e(t)\|^{2} \\
& +\|\hat{e}(t)\|^{2}+\left\|\hat{e}_{x}(t)\right\|^{2}+\tilde{D}(t)^{2}
\end{aligned}
$$

There exists $\gamma^{*}>0, R>0$ and $\rho>0$ such that, if the initial state satisfies $\Gamma(0)<\rho$ and if $0 \leq \gamma_{D}<\gamma^{*}$ then

$$
\begin{gathered}
\forall t \geq 0, \quad \Gamma(t) \leq R \Gamma(0) \\
Y(t) \underset{t \rightarrow \infty}{\rightarrow} Y^{r}, \quad X(t) \underset{t \rightarrow \infty}{\longrightarrow} X^{r}, \quad \tilde{X}(t) \underset{t \rightarrow \infty}{\rightarrow} 0 \\
\text { and } \quad U(t) \underset{t \rightarrow \infty}{\rightarrow} U^{r}
\end{gathered}
$$

The state variables of the plant (5) and the observer (7) are $(X, \hat{X}, u, \hat{u}, \hat{D})$. Equivalently, the condition stated in Theorem 1 $(\Gamma(0)$ sufficiently small) requires that, initially, each of these variables are sufficiently close to their corresponding trajectories (namely $X^{r}, U^{r}$ and the unknown value $D$ ). Another formulation is presented in the Lyapunov proof in the next section, as this condition is expressed with the equivalent variables $(\Delta \hat{X}, \tilde{X}, \tilde{e}, \hat{w})(\operatorname{see}(22))$.

\section{PROOF OF THEOREM 1}

In the following, we use the systematic Lyapunov tools introduced by Krstic [2008] to analyze the stability of input timedelay systems. These tools are based on a backstepping transformation of the actuator state ${ }^{1}$. Pursuing this approach, we define the following transformed distributed input, satisfying a Volterra integral equation of the second kind,

$$
\begin{aligned}
\hat{w}(x, t)= & \hat{e}(x, t)-\hat{D}(t) \int_{0}^{x} K e^{A \hat{D}(t)(x-y)} B \hat{e}(y, t) d y \\
& -K e^{A \hat{D}(t) x} \Delta \hat{X}(t)
\end{aligned}
$$

\footnotetext{
1 this transformation is made to convert the plant (5) into the target system $\dot{X}(t)=(A+B K) X(t)+B w(0, t), Y(t)=C X(t)$

$D w_{t}(x, t)=w_{x}(x, t)$ with the boundary condition $w(1, t)=0$
} 
jointly with its inverse,

$$
\begin{aligned}
\hat{e}(x, t)= & \hat{w}(x, t)+\hat{D}(t) \int_{0}^{x} K e^{(A+B K) \hat{D}(t)(x-y)} B \hat{w}(y, t) d y \\
& +K e^{(A+B K) \hat{D}(t) x} \Delta \hat{X}(t)
\end{aligned}
$$

designed to fulfill $\hat{w}(1, t)=0$.

\subsection{Lyapunov analysis}

We introduce the following Lyapunov-Krasovskii functional candidate

$$
\begin{aligned}
V(t)= & \Delta \hat{X}(t)^{T} P_{1} \Delta \hat{X}(t)+b_{0} \tilde{X}(t)^{T} P_{2} \tilde{X}(t) \\
& +b_{1} D \int_{0}^{1}(1+x) \tilde{e}(x, t)^{2} d x \\
& +b_{2} \hat{D}(t) \int_{0}^{1}(1+x) \hat{w}(x, t)^{2} d x \\
& +b_{2} \hat{D}(t) \int_{0}^{1}(1+x) \hat{w}_{x}(x, t)^{2} d x+\tilde{D}(t)^{2}
\end{aligned}
$$

where $b_{0}, b_{1}$ and $b_{2}$ are positive coefficients and the symmetric definite matrices $P_{1}$ and $P_{2}$ satisfy the following Lyapunov equations, for given symmetric definite positive matrices $Q_{1}$ and $Q_{2}$,

$$
\begin{aligned}
P_{1}(A+B K)+(A+B K)^{T} P_{1} & =-Q_{1} \\
P_{2}(A+L C)+(A+L C)^{T} P_{2} & =-Q_{2}
\end{aligned}
$$

This functional is equivalent to $\Gamma$, defined in (18), as will be shown, but reveals more helpful in the analysis, as the set of variables $\left(\tilde{e}, \hat{w}, \hat{w}_{x}\right)$ presents boundary conditions equal to zero. In details, this particular property is handy through integrations by parts, involving the factor $(1+x)$ under the integrals, to create bounding negative terms.

First, consider the dynamics of the variables involved in (24), which can be written, using (22) and (23), as

$$
\begin{aligned}
& \dot{\tilde{X}}(t)=(A+L C) \tilde{X}(t)+B \tilde{e}(0, t) \\
& \frac{d \Delta \hat{X}}{d t}=(A+B K) \Delta \hat{X}(t)+B \hat{w}(0, t)-L C \tilde{X}(t) \\
& D \tilde{e}(x, t)=\tilde{e}_{x}(x, t)-\tilde{D}(t) g(x, t) \\
&-\hat{\hat{D}}(t) D(x-1) g(x, t) \\
& \tilde{e}(1, t)=0 \\
& \hat{D}(t) \hat{w}_{t}(x, t)=\hat{w}_{x}(x, t)-\hat{\hat{D}}(t) \hat{D}(t) h(x, t) \\
& \quad+\hat{D}(t) K e^{A \hat{D}(t) x} L C \tilde{X}(t) \\
& \hat{w}(1, t)=0 \\
& \hat{D}(t) \hat{w}_{x t}(x, t)=\hat{w}_{x x}(x, t)-\dot{\hat{D}}(t) \hat{D}(t) h_{x}(x, t) \\
& \quad+\hat{D}(t)^{2} K A e^{A \hat{D}(t) x} L C \tilde{X}(t) \\
& \hat{w}_{x}(1, t)=\hat{\hat{D}}(t) \hat{D}(t) h(1, t)-\hat{D}(t) K e^{A \hat{D}(t)} L C \tilde{X}(t)
\end{aligned}
$$

where the functions $g$ and $h$ are defined in Appendix A. Taking a time-derivative of $V$ and using suitable integrations by parts, we obtain

$$
\begin{aligned}
\dot{V}(t)= & -\Delta \hat{X}(t)^{T} Q_{1} \Delta \hat{X}(t)+2 \Delta \hat{X}(t)^{T} P_{1} B \hat{w}(0, t) \\
& -2 \Delta \hat{X}(t)^{T} P_{1} L C \tilde{X}(t)+b_{0}\left(-\tilde{X}(t)^{T} Q_{2} \tilde{X}(t)\right. \\
& \left.+2 \tilde{X}(t) P_{2} B \tilde{e}(0, t)\right)+b_{1}\left(-\|\tilde{e}(t)\|^{2}-\tilde{e}(0, t)^{2}\right. \\
& -2 \tilde{D}(t) \int_{0}^{1}(1+x) \tilde{e}(x, t) g(x, t) d x \\
& \left.+2 \dot{\hat{D}}(t) D \int_{0}^{1}\left(1-x^{2}\right) \tilde{e}(x, t) g(x, t) d x\right)
\end{aligned}
$$

$$
\begin{aligned}
& +b_{2}\left(-\|\hat{w}(t)\|^{2}-\hat{w}(0, t)^{2}\right. \\
& -2 \dot{\hat{D}}(t) \hat{D}(t) \int_{0}^{1}(1+x) \hat{w}(x, t) h(x, t) d x \\
& \left.+2 \hat{D}(t) \int_{0}^{1}(1+x) \hat{w}(x, t) K e^{A \hat{D}(t) x} L C \tilde{X}(t) d x\right) \\
& +b_{2}\left(2 \hat{w}_{x}(1, t)^{2}-\hat{w}_{x}(0, t)^{2}-\left\|\hat{w}_{x}(t)\right\|^{2}\right. \\
& -2 \hat{D}(t) \dot{\hat{D}}(t) \int_{0}^{1}(1+x) \hat{w}_{x}(x, t) h_{x}(x, t) d x \\
& \left.+2 \hat{D}(t)^{2} \int_{0}^{1}(1+x) \hat{w}_{x}(x, t) K A e^{A \hat{D}(t) x} L C \tilde{X}(t) d x\right) \\
& +b_{2} \dot{\hat{D}}(t)\left(\int_{0}^{1}(1+x) \hat{w}(x, t)^{2} d x\right. \\
& \left.+\int_{0}^{1}(1+x) \hat{w}_{x}(x, t)^{2} d x\right)-2 \tilde{D}(t) \dot{\hat{D}}(t)
\end{aligned}
$$

Choosing $b_{2} \geq 4\left|P_{1} B\right|^{2} / \lambda_{1}, b_{0} \geq 16 \frac{\left|P_{2} L C\right|^{2}}{\lambda_{1} \lambda_{2}}$ and applying Young's inequality with Assumption 4, one gets

$$
\begin{aligned}
\dot{V}(t) & \leq-\frac{\lambda_{1}}{4}|\Delta \hat{X}(t)|^{2}-\frac{b_{0} \lambda_{2}}{4}|\tilde{X}(t)|^{2}-b_{1}\|\tilde{e}(t)\|^{2} \\
& -\left(b_{1}-\frac{2\left|P_{2} B\right|^{2}}{\lambda_{2} b_{0}}\right) \tilde{e}(0, t)^{2}-b_{2}\|\hat{w}(t)\|^{2} \\
& -\frac{b_{2}}{2} \hat{w}(0, t)^{2}+2 b_{2} \hat{w}_{x}(1, t)^{2}-b_{2}\left\|\hat{w}_{x}\right\|^{2} \\
& -b_{2} \hat{w}_{x}(0, t)^{2}+2 b_{1}|\tilde{D}(t)| \int_{0}^{1}(1+x) \mid \tilde{e}(x, t) \| g(x, t \mid d x \\
& +2 b_{1} \gamma_{D} M D \int_{0}^{1}\left(1-x^{2}\right)|\tilde{e}(x, t) \| g(x, t)| d x \\
& +2 b_{2} \gamma_{D} M \hat{D}(t) \int_{0}^{1}(1+x)|\hat{w}(x, t) \| h(x, t)| d x \\
& +2 b_{2} \hat{D}(t) \int_{0}^{1}(1+x)\left|K e^{A \hat{D}(t) x} L C \tilde{X}(t) \hat{w}(x, t)\right| d x \\
& +2 b_{2} \gamma_{D} M \hat{D}(t)\left|\int_{0}^{1}(1+x) \hat{w}_{x}(x, t) h_{x}(x, t) d x\right| \\
& +2 b_{2} \hat{D}(t)^{2} \int_{0}^{1}(1+x)\left|K A e^{A \hat{D}(t) x} L C \tilde{X}(t) \hat{w}_{x}(x, t)\right| d x \\
& +2 b_{2} \gamma_{D} M\left(\|\hat{w}(t)\|^{2}+\left\|\hat{w}_{x}(t)\right\|^{2}\right)
\end{aligned}
$$

We now use the inequalities given in Appendix B to bound the resulting positive terms. By choosing $b_{1} \geq \frac{2\left|P_{2} B\right|^{2}}{\lambda_{2} b_{0}}$ and $b_{0} \geq$ $\frac{8 b_{2}}{\lambda_{2}}\left(M_{3}+M_{4}+M_{6}\right)$, we can define the following quantities

$$
\begin{aligned}
V_{0}(t) & =|\Delta \hat{X}(t)|^{2}+|\tilde{X}(t)|^{2}+\|\tilde{e}(t)\|^{2}+\|\hat{w}(t)\|^{2}+\left\|\hat{w}_{x}(t)\right\|^{2} \\
\eta & =\min \left\{\lambda_{1} / 4, b_{0} \lambda_{2} / 8, b_{1}, b_{2} / 2\right\}
\end{aligned}
$$

and obtain

$$
\begin{aligned}
\dot{V}(t) \leq & -\left(\eta-b_{1} M_{1}|\tilde{D}(t)|-\gamma_{D}\left(b_{1} M M_{1}+b_{2} M M_{2}\right.\right. \\
& \left.\left.+b_{2} \gamma_{D} M_{4}+b_{2} M M_{5}+2 b_{2} M\right)\right) V_{0}(t) \\
& -b_{2}\left(1-\gamma_{D} M\right) \hat{w}_{x}(0, t)^{2}
\end{aligned}
$$

Employing the bound $|\tilde{D}(t)| \leq \frac{\varepsilon}{2}+\frac{V(t)}{2 \varepsilon}$, with $\varepsilon>0$, and defining $m\left(\gamma_{D}\right)=b_{1} M M_{1}+b_{2} M M_{2}+b_{2} M M_{5}+2 b_{2} M+b_{2} \gamma_{D} M_{4}$, we get 


$$
\begin{aligned}
\dot{V}(t) \leq & -\left(\eta-b_{1} M_{1}\left[\frac{\varepsilon}{2}+\frac{V(t)}{2 \varepsilon}\right]-\gamma_{D} m\left(\gamma_{D}\right)\right) V_{0}(t) \\
& -b_{2}\left(1-\gamma_{D} M\right) \hat{w}_{x}(0, t)^{2}
\end{aligned}
$$

Then, choosing the parameter $\varepsilon$ and the gain $\gamma_{D}$ such that

$$
\begin{aligned}
\varepsilon & <\frac{2 \eta}{b_{1} M_{1}} \\
\gamma_{D} & <\min \left\{\frac{1}{M}, 1, \frac{\eta-b_{1} M_{1} \varepsilon / 2}{m(1)}\right\}
\end{aligned}
$$

and restricting the initial condition so that

$$
V(0)<2 \varepsilon \frac{\eta-b_{1} M_{1} \varepsilon / 2-\gamma_{D} m\left(\gamma_{D}\right)}{b_{1} M_{1}}
$$

we finally conclude that there exists a non-negative function $\mu$ such that

$$
\dot{V}(t) \leq-\mu(t) V_{0}(t)
$$

Therefore, in the end, we have established that

$$
\forall t \geq 0, \quad V(t) \leq V(0)
$$

\subsection{Equivalence}

In view of obtaining the asymptotic stability result stated in Theorem 1, we prove here that the two functionals $\Gamma$ and $V$ are equivalent, i.e. that there exist constants $a>0$ and $b>0$ such that $a V(t) \leq \Gamma(t) \leq b V(t)$.

First, considering (22)-(23) and applying Young's inequality, we establish the following inequalities

$$
\begin{aligned}
\|\hat{e}(x, t)\|^{2} & \leq r_{1}\|\hat{w}(x, t)\|^{2}+r_{2}|\Delta \hat{X}(t)|^{2} \\
\left\|\hat{e}_{x}(x, t)\right\|^{2} & \leq r_{3}\left\|\hat{w}_{x}(x, t)\right\|^{2}+r_{4}\|\hat{w}(x, t)\|^{2}+r_{5}|\Delta \hat{X}(t)|^{2} \\
\|\hat{w}(x, t)\|^{2} & \leq s_{1}\|\hat{e}(x, t)\|^{2}+s_{2}\|\Delta \hat{X}(t)\|^{2} \\
\left\|\hat{w}_{x}(x, t)\right\|^{2} & \leq s_{3}\left\|\hat{e}_{x}(x, t)\right\|^{2}+s_{4}\|\hat{e}(x, t)\|^{2}+s_{5}|\Delta \hat{X}(t)|^{2}
\end{aligned}
$$

where $r_{1}, r_{2}, r_{3}, r_{4}, r_{5}, s_{1}, s_{2}, s_{3}, s_{4}$ and $s_{5}$ are positive constants. Then, one easily obtains

$$
\begin{aligned}
\Gamma_{3}(t) \leq & 2|\tilde{X}(t)|^{2}+2|\Delta \hat{X}(t)|^{2}+2\|\tilde{e}(t)\|^{2}+3\|\hat{e}(t)\|^{2} \\
& +\left\|\hat{e}_{x}(t)\right\|^{2}+\tilde{D}(t)^{2} \\
\leq & \frac{\max \left\{3+3 r_{2}+r_{5}, 3 r_{1}+r_{4}, r_{3}\right\}}{\min \left\{\lambda_{1}, b_{0} \lambda_{2}, b_{1} D, b_{2} \underline{D}, 1\right\}} V_{3}(t) \\
V_{3}(t) \leq & \max \left\{\lambda_{1}+2 b_{0} \lambda_{2}+2 b_{2} \bar{D}\left(s_{2}+s_{5}\right),\right. \\
& \left.4 b_{1} D+2 b_{2} \bar{D}\left(s-1+s_{4}\right), 2 b_{2} \bar{D} s_{3}, 1\right\} \Gamma(t)
\end{aligned}
$$

which gives the desired stability result (19) with $R=b / a$.

\subsection{Convergence properties}

We now conclude the proof of Theorem 1, applying Barbalat's Lemma for $|\tilde{X}(t)|^{2},|\Delta \hat{X}(t)|^{2}$ and $\tilde{U}(t)^{2}$.

Integrating (39) between 0 and $+\infty$, it is easy to obtain the integrability of the first two quantities. Further, from (27)-(28), we get

$$
\frac{d|\tilde{X}(t)|^{2}}{d t}=2 \tilde{X}(t)^{T}((A+L C) \tilde{X}(t)+B \tilde{e}(0, t))
$$

$\frac{d}{d t}\left(|\Delta \hat{X}(t)|^{2}\right)=2 \Delta \hat{X}(t)^{T}((A+B K) \Delta \hat{X}(t)+B \hat{w}(0, t)-L C \tilde{X}(t))$

From (40), it follows that $|\tilde{X}(t)|,|\Delta \hat{X}(t)|,\|\tilde{e}(t)\|,\|\hat{w}(t)\|$ and $\left\|\hat{w}_{x}(t)\right\|$ are uniformly bounded. Then, with (41), we obtain the uniform boundedness of $\|\hat{e}(t)\|$ and, consequently, of $\|\hat{u}(t)\|$. With (9), we conclude that $U(t)$ is uniformly bounded, and, therefore, that $\tilde{e}(0, t)=U(t-D)-U(t-\hat{D}(t))$ is bounded for $t \geq \bar{D}$. We finally get the boundedness of $d\left(|\tilde{X}(t)|^{2}\right) / d t$ for $t \geq \bar{D}$ and conclude, with Barbalat's Lemma, that $\tilde{X}(t) \rightarrow 0$ as $t \rightarrow \infty$.

Similarly, from the definition (22), we get the boundedness of $\hat{w}(0, t)$ for $t \geq \bar{D}$ and, immediately, the one of $d\left(|\Delta \hat{X}(t)|^{2}\right) / d t$ for $t \geq \bar{D}$. We conclude, by Barbalat's Lemma, that $\Delta \hat{X}(t) \rightarrow 0$ as $t \rightarrow \infty$. Comparing this result with the previous one, we get (20).

Finally, one can obtain

$$
\frac{d \tilde{U}(t)^{2}}{d t}=2 \tilde{U}(t)\left(K e^{A \hat{D}(t)} \frac{d(\Delta \hat{X}(t))}{d t}+\dot{\hat{D}}(t) G_{0}(t)+H_{0}(t)\right)
$$

with

$$
\begin{aligned}
G_{0}(t)= & K\left[e^{A \hat{D}(t)} A \Delta \hat{X}(t)\right. \\
& +\int_{0}^{1}(I+A \hat{D}(t)(1-y)) e^{A \hat{D}(t)(1-y)} B \hat{e}(y, t) d y \\
& \left.+\int_{0}^{1} e^{A \hat{D}(t)(1-y)} B(y-1) \hat{e}_{x}(y, t) d y\right] \\
H_{0}(t)= & K \int_{0}^{1} e^{A \hat{D}(t)(1-y)} B \hat{e}_{x}(y, t) d y
\end{aligned}
$$

From the previous analysis, it is straightforward to obtain the uniform boundedness of $G_{0}(t)$ and $H_{0}(t)$. Then, with Assumption 4, we get the uniform boundedness of $d \tilde{U}(t)^{2} / d t$ and we conclude by Barbalat's Lemma.

\section{ILLUSTRATIVE EXAMPLE}

In this section, to illustrate the merits and the feasibility of the proposed approach, we consider an open-loop unstable process of order three with an unknown time-delay studied in Huang and Lin [1995] and Huang and Chen [1997]

$$
Y(s)=\frac{e^{-0.5 s}}{(5 s-1)(2 s+1)(0.5 s+1)} U(s)
$$

We compare the obtained simulation results to both the PID proposed in Huang and Lin [1995] and the three-elements structure proposed by Huang and Chen [1997]. The general scheme of our strategy is given in Fig. 1

\subsection{Model and necessity of an observer}

In Huang and Chen [1997], the proposed controller is designed only for first-order and second-order delayed unstable plants. Consequently, for control purposes, the author use the effective following approximation

$$
Y(s) \approx \frac{e^{-0.939 s}}{(5 s-1)(2.07 s+1)} U(s)=\frac{e^{-D s}}{(a s-1)(T s+1)} U(s)
$$

proposed in Huang and Lin [1995]. In details, the stable part of the transfer function has been approximated by a first-order delayed function, using a closed-loop identification technique and a least mean-square optimization in the frequency domain.

The controller settings are sought after based on this approximation model, which highlights the necessity of constructing an observer. We introduce the following state-space representation

$$
A=\left(\begin{array}{cc}
0 & \frac{1}{5 \times 2.07} \\
1 & {\left[\frac{1}{5}-\frac{1}{2.07}\right]}
\end{array}\right), B=\left(\begin{array}{c}
\frac{1}{5} \times \frac{1}{2.07} \\
0
\end{array}\right), C=\left(\begin{array}{ll}
0 & 1
\end{array}\right)
$$




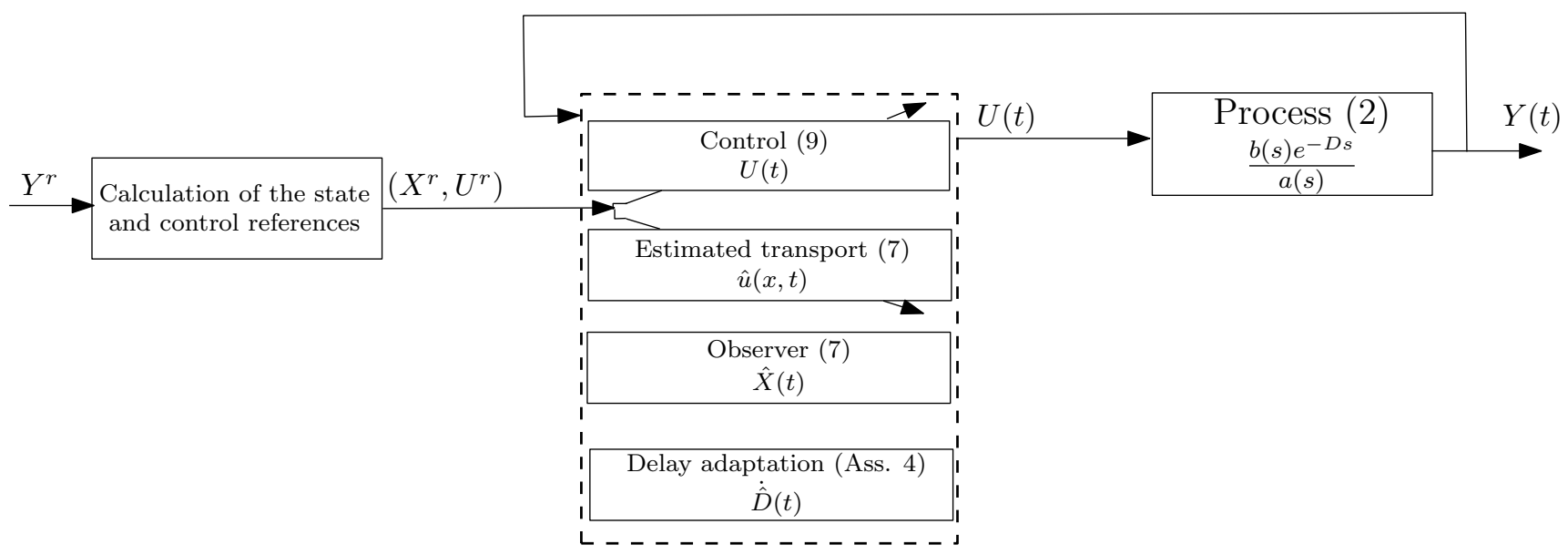

Fig. 1. The proposed adaptive control structure. The closed-loop algorithm uses distributed parameters system (i.e a varying speed waiting-line) and a system state observer.

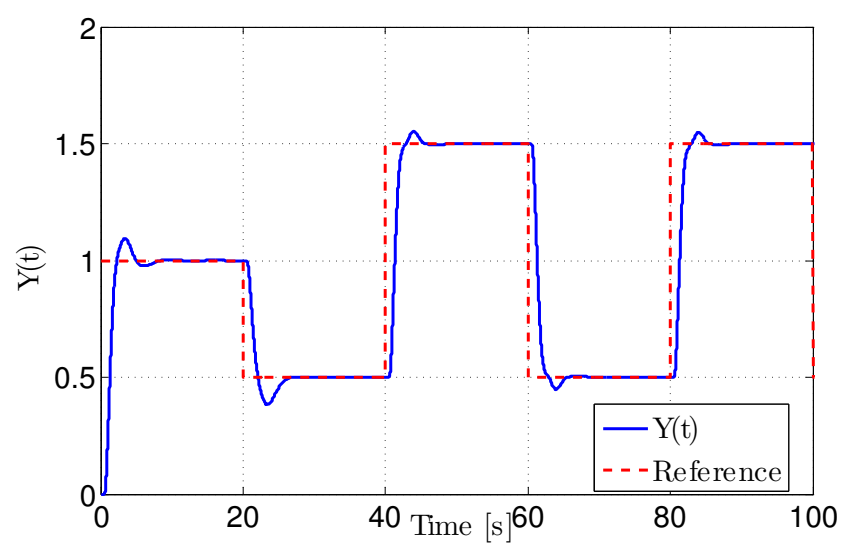

(a) $\mathrm{Y}(\mathrm{t})$

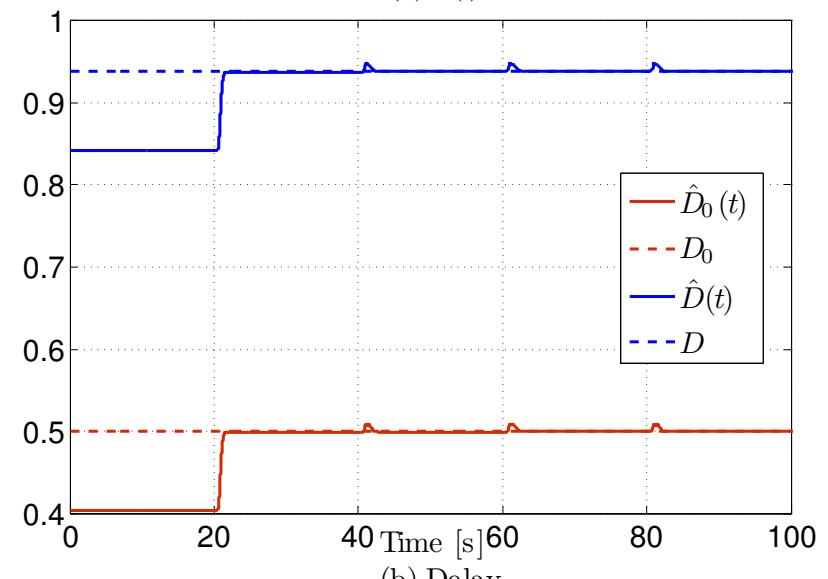

(b) Delay

Fig. 2. Response of the adaptive controller for the output trajectory presented in Fig. 2(a), with $Y(0)=0, u(:, 0)=0$ and $\hat{D}_{0}(0)=0.4 s$ and with the delay adaptation strategy presented in Section 5.

and construct the observer

$$
\begin{cases}\dot{\hat{X}}(t) & =A \hat{X}(t)+B \hat{u}(0, t)+L(Y-C \hat{X}(t)) \\ \hat{D}(t) u_{t}(x, t) & =\hat{u}_{x}(x, t)+\hat{\hat{D}}(t)(x-1) \hat{u}_{x}(x, t) \\ \hat{u}(1, t) & =U(t)\end{cases}
$$

where the observer gain are, e.g. set to $L=\left[\begin{array}{ll}-2.09 & -2.72\end{array}\right]^{T}$.

\subsection{Delay adaptation}

To obtain a delay update law satisfying Assumption 4, we consider here the following instantaneous cost function

$$
\phi:(t, \hat{D}) \in\left[t_{0} ;+\infty\right] \times[\underline{D} ; \bar{D}] \mapsto\left|Y_{P}(t, \hat{D})-Y(t)\right|^{2}
$$

where $Y_{p}(t, \hat{D})$ is a $\left(t-t_{0}\right)$-units of time prediction of the system output, starting from $Y\left(t_{0}\right)$ as initial condition and assuming that the actual delay value is $\hat{D}(t)$. The instant $t_{0}$ is chosen such that the corresponding system state $X\left(t_{0}\right)$ is known. Then, using a steepest descent algorithm, one can take

$$
\tau_{D}(t)=-\gamma_{D}\left(Y_{P}(t, \hat{D})-Y(t)\right) \times \frac{\partial Y_{P}}{\partial \hat{D}}(t, \hat{D})
$$

This choice is based on the same idea that in most identification techniques, i.e. the comparison of two versions of the output signal, the one corresponding to the unknown delay $D$ and the other to the controlled delay $\hat{D}(t)$.

This adaptation gives an accurate estimation of the unknown delay, provided that the initial delay estimate is sufficiently close to the true delay value. In details, this condition, which is compliant with the one already stated in the Lyapunov analysis, guarantees that no extraneous local minimum interfere with the minimization process.

The instable part of the process favorably conditions the problem, but requires cautious discretizations while simulating the process. Further, the computation of $Y_{P}$, and particularly of the involved integral, requires an accurate knowledge of the dynamics of the system. For this reason, we define the following state-space representation of the original transfer function (44), jointly with the original delay $D_{0}=0.5 \mathrm{~s}$,

$$
\begin{aligned}
A_{0} & =\left(\begin{array}{ccc}
0 & 1 & 0 \\
0 & 0 & 1 \\
0.2 & -0.5 & -2.3
\end{array}\right) \quad, \quad B_{0}=\left(\begin{array}{c}
0 \\
0 \\
0.2
\end{array}\right) \\
C_{0} & =\left(\begin{array}{lll}
1 & 0 & 0
\end{array}\right)
\end{aligned}
$$

The two delays are related by by the following expression $D(t)=D_{0}(t)+0.439$, that we extend to their estimations. With this relation, the signals $Y_{P}$ and $\partial Y_{P} / \partial \hat{D}$ can be computed via the exact dynamics of the system output as 


\begin{tabular}{|c|c|c|}
\hline & IAE [-] & $T_{5 \%}[\mathrm{~s}]$ \\
\hline Proposed Strategy & 1.55 & 3.04 \\
\hline Three-element Controller & 3.04 & 8.02 \\
\hline PID & 5.37 & 14.81 \\
\hline
\end{tabular}

Table 1. Performances in tracking for the first reference step (0-20s) for the different controllers: Integral Absolute Error (IAE) and 5\% time-response.

$$
Y_{P}(t, \hat{D})=C_{0}\left[e^{A_{0}\left(t-t_{0}\right)} X\left(t_{0}\right)+\int_{t_{0}}^{t} e^{A_{0}(t-\tau)} B_{0} U\left(\tau-\hat{D}_{0}\right) d \tau\right]
$$

$\frac{\partial Y_{P}}{\partial \hat{D}}(t, \hat{D})=-C_{0} \int_{t_{0}}^{t} e^{A_{0}(t-\tau)} B_{0} \frac{d U}{d t}\left(\tau-\hat{D}_{0}\right) d \tau$

We can deduce from this adaptation the estimate $\hat{D}(t)=$ $\hat{D}_{0}(t)+0.439$ and use it in the control.

\subsection{Simulation set-up and results}

The tracked trajectory is given in Fig. 2(a). The configuration of the first 20s in the simulation are similar to the one presented in Huang and Chen [1997], with zero initial conditions $Y(0)=0$ and $u(:, 0)=0=\hat{u}(:, 0)$. The initial delays estimates are taken as $\hat{D}_{0}(0)=0.4=\hat{D}(0)-0.439$. Finally, the control gain $K$ is chosen thanks to a LQR criterion, and the delay update gain is arbitrarily set to $\gamma_{D}=10$.

The obtained results are presented in Fig.2. First, as shown in Table 1, the proposed strategy compares favorably to the two other controllers mentioned in this paper, in both accuracy of tracking and speed. This conclusion is not surprising as the reference controller of Huang and Chen [1997] is set by considering a certain trade-off with other criteria, such as the ability to reject disturbances which are out-of-the-scope of this paper.

Second, one can notice on Fig.2(b) that the delay estimate provides a good identification of the unknown delay. The most visible improvements in this estimation occur at step changes of the reference signal. This is consistent with the employed update law, as the cost function presents its most important gradient at these instants. In other words, the transient behaviors of the system output provides delay observability.

The delay identification has direct consequences on the control efficiency : overshoots observed in the output for each reference step change are less important after $40 s$, which correspond to the convergence of the delay estimate.

\section{CONCLUSION}

In this paper, a state observer technique has been proposed to control time-delay system, with an uncertainty on the delay, through the methodology introduced in Krstic [2008], and further developed in view of application in Bresch-Pietri et al. [2010]. This extension is of particular interest for systems given under the form of a transfer function, such as the example chosen to illustrate the method. Such SISO descriptions are generalized in numerous process-control application, where step tests are used to identify the various influences of the inputs of the systems (sse e.g. Richalet [1998] and Petit et al. [2002]).

One path to explore is the application of the presented approach to such systems. Other problem of importance, both from a practical and a theoretical view points, is the issue of disturbance rejection. Combining integrators with the presented methodology seems like a natural solution to reject biases but a convergence analysis will certainly require careful attention. A third issue is the robustness to plant uncertainties, for which the incorporation of the adaptive scheme developed in BreschPietri et al. [2010] into the presented control seems a promising way. These are subjects of future research.

\section{APPENDIX}

\subsection{Appendix A : Dynamics functions}

$$
\begin{aligned}
& g(x, t)=\frac{\hat{w}_{x}(x, t)}{\hat{D}(t)}+K B \hat{w}(x, t)+\hat{D}(t) \int_{0}^{x} K(A+B K) \\
& \quad \times e^{(A+B K) \hat{D}(t)(x-y)} B \hat{w}(y, t) d y \\
& \quad+K(A+B K) e^{(A+B K) \hat{D}(t) x} \Delta \hat{X}(t) \\
& h(x, t)=(1-x) g(x, t)+\hat{D}(t) \int_{0}^{x} K e^{A \hat{D}(t)(x-y)} B(y-1) \\
& \quad \times g(y, t) d y+\int_{0}^{x} K(I+A \hat{D}(t)(x-y)) e^{A \hat{D}(t)(x-y)} B \\
& \quad\left[\hat{w}(y, t)+\hat{D}(t) \int_{0}^{y} K e^{(A+B K) \hat{D}(t)(y-\xi)} B \hat{w}(\xi, t) d \xi\right. \\
& \left.+K e^{(A+B K) \hat{D}(t) y} \Delta \hat{X}(t)\right] d y+K A x e^{A \hat{D}(t) x} \Delta \hat{X}(t)
\end{aligned}
$$

\subsection{Appendix B : Bounds used in the Lyapunov Analysis}

Using the expression of the functions given in Appendix A, jointly with Young's inequality and Cauchy-Schwartz's inequality, one can show that there exist positive constants $M_{1}, M_{2}, \ldots, M_{6}$ independent of initial conditions such that

$$
\begin{aligned}
& 2 \int_{0}^{1}(1+x)|\tilde{e}(x, t)||g(x, t)| d x \\
& \leq M_{1}\left(|\Delta \hat{X}(t)|^{2}+\|\tilde{e}(t)\|^{2}+\|\hat{w}(t)\|^{2}+\left\|\hat{w}_{x}(t)\right\|^{2}\right) \\
& 2 D \int_{0}^{1}\left(1-x^{2}\right)|\tilde{e}(x, t)||g(x, t)| d x \\
& \leq M_{1}\left(|\Delta \hat{X}(t)|^{2}+\|\tilde{e}(t)\|^{2}+\|\hat{w}(t)\|^{2}+\left\|\hat{w}_{x}(t)\right\|^{2}\right) \\
& 2 \hat{D}(t) \int_{0}^{1}(1+x)|\hat{w}(x, t)||h(x, t)| d x \\
& \leq M_{2}\left(|\Delta \hat{X}(t)|^{2}+\|\hat{w}(t)\|^{2}+\left\|\hat{w}_{x}(t)\right\|^{2}\right) \\
& 2 \hat{D}(t) \int_{0}^{1}(1+x)\left|K e^{A \hat{D}(t) x} L C \hat{w}(x, t) d x\right| \\
& \leq M_{3}|\tilde{X}(t)|^{2}+\|\hat{w}(t)\|^{2} / 2 \\
& 2 \hat{w}_{x}(1, t)^{2} \\
& \leq M_{4}\left(|\tilde{X}(t)|^{2}+\gamma_{D}^{2}\left(|\Delta \hat{X}(t)|^{2}+\|\hat{w}(t)\|^{2}+\left\|\hat{w}_{x}(t)\right\|^{2}\right)\right) \\
& 2 \hat{D}(t)\left|\int_{0}^{1}(1+x) \hat{w}_{x}(x, t) h(x, t) d x\right| \\
& \leq \hat{w}_{x}(0, t)^{2}+M_{5}\left(|\Delta \hat{X}(t)|^{2}+\|\hat{w}(t)\|^{2}+\left\|\hat{w}_{x}(t)\right\|^{2}\right) \\
& 2 \hat{D}(t)^{2} \int_{0}^{1}(1+x)\left|K A e^{A \hat{D} x} L C \hat{w}_{x}(x, t)\right| d x \\
& \leq M_{6}|\tilde{X}(t)|^{2}+\|\hat{w}(t)\|^{2} / 2
\end{aligned}
$$




\section{REFERENCES}

Z. Artstein. Linear systems with delayed controls: a reduction. IEEE Transactions on Automatic Control, 27(4):869-879, 1982.

D. Bresch-Pietri and M. Krstic. Adaptive trajectory tracking despite unknown input delay and plant parameters. Automatica, 45(9):2074-2081, 2009.

D. Bresch-Pietri, J. Chauvin, and N. Petit. Adaptive backstepping controller for uncertain systems with unknown input time-delay. application to si engines. Proceedings of the 2010 Conference on Decision and Control, 2010.

C. T. Huang and Y. S. Lin. Tuning PID controller for open-loop unstable processes with time delay. Chemical Engineering Communications, 133(1):11-30, 1995.

H.P. Huang and C.C. Chen. Control-system synthesis for openloop unstable process with time delay. IEE Proc-Control Theory Appl, 144(4), 1997.

T. Kailath. Linear systems. Prentice-Hall Englewood Cliffs, NJ, 1980.

M. Krstic. Boundary control of PDEs: A course on backstepping designs. Society for Industrial and Applied Mathematics Philadelphia, PA, USA, 2008.

M. Krstic and D. Bresch-Pietri. Delay-adaptive full-state predictor feedback for systems with unknown long actuator delay. In Proceedings of the 2009 conference on American Control Conference, pages 4500-4505, 2009.

M. Krstic and A. Smyshlyaev. Backstepping boundary control for first-order hyperbolic PDEs and application to systems with actuator and sensor delays. Systems \& Control Letters, 57(9):750-758, 2008.

A. O'Dwyer. A survey of techniques for the estimation and compensation of processes with time delay. Report $N^{o} A O D$. 00.03, Dublin Institute of Technology, Irlanda, 2000.

N. Petit, Y. Creff, and P. Rouchon. Motion planning for two classes of nonlinear systems with delays depending on the control. 1:1007-1011, 2002.

J. Richalet. Pratique de l'identification. Hermes, 1998.

J.-P. Richard. Time-delay systems: an overview of some recent advances and open problems. Automatica, 39(10):16671694, 2003.

O. J. M. Smith. A controller to overcome dead time. ISA Journal, 6(2):28-33, 1959.

Q. C. Zhong. Robust control of time-delay systems. Springer Verlag, 2006. 